\title{
Downregulation of Ezh2 expression by RNA interference induces cell cycle arrest in the G0/G1 phase and apoptosis in U87 human glioma cells
}

\author{
RUIJIAN ZHANG ${ }^{1 *}$, RUIJUN WANG ${ }^{2 *}$, HONG CHANG $^{1}$, FEI WU ${ }^{3}$, \\ CHUNTAO LIU ${ }^{3}$, DONGFENG DENG ${ }^{3}$ and WENHAI FAN ${ }^{3}$ \\ ${ }^{1}$ Department of Neurosurgery, Inner Mongolia People's Hospital, Hohhot; ${ }^{2}$ First Affiliated Hospital of Inner \\ Mongolia Medical College, Hohhot; ${ }^{3}$ Affiliated Zhongshan Hospital of Dalian University, Dalian, P.R. China
}

Received May 31, 2012; Accepted August 17, 2012

DOI: $10.3892 /$ or.2012.2033

\begin{abstract}
The Ezh2 gene is an important member of the polycomb-group (PcG) family. As a newly identified oncogene, the expression of Ezh2 has been shown to be significantly increased in prostate cancer, breast cancer, renal cell carcinoma and hepatic cancer; however, a role for Ezh2 in the occurrence of glioma has not yet been reported. In this study, we found that the Ezh2 gene is highly expressed in U87 human glioma cells. Using RNA interference, we demonstrated that the downregulation of Ezh2 expression in U87 human glioma cells resulted in apoptosis and a cell cycle arrest in the G0/G1 phase. In addition, we found that silencing of the Ezh2 gene altered the mitochondrial membrane potential and promoted the release of cytochrome $c$ from the mitochondria. Furthermore, the reduced expression of Ezh2 altered the Bax and Bcl-2 protein levels and led to the activation of caspase 9 and 3. These results indicate that the apoptosis induced in U87 human glioma cells by the silencing of the Ezh2 gene is related to the mitochondrial pathway.
\end{abstract}

\section{Introduction}

Glioma is the most common type of primary tumour found in the central nervous system, where it accounts for $45-55 \%$ of all primary tumours and is a leading cause of mortality in patients with intracranial tumours $(1,2)$. Currently, conventional treatments for glioma include surgery, radiotherapy, and chemotherapy $(3,4)$; however, the efficacy of these treatments remains poor. In addition, the molecular mechanisms that

Correspondence to: Dr Wenhai Fan, Affiliated Zhongshan Hospital of Dalian University, 6 Jiefang Street, Zhongshan District, Dalian, Liaoning 116001, P.R. China

E-mail:whffwh@tom.com

${ }^{*}$ Contributed equally

Key words: Ezh2, glioma, RNA interference, apoptosis, mitochondria result in the occurrence and development of glioma are not yet well understood. Therefore, the discovery of molecules with roles in the occurrence and development of glioma is required to understand the malignant biological behaviour of glioma. These novel molecules may provide valuable, reliable molecular targets for future targeted therapies.

The Ezh2 gene is an important member of the polycombgroup (PcG) family. PcG genes regulate the transcription process and thereby play an important role in the regulation of cell proliferation and the cell cycle. The Ezh2 gene is often expressed at low levels in normal cells but at high levels in a variety of stem cells (5-7). In addition, expression of the Ezh2 gene has been found in many tumour cells, including hepatic cancer (8), breast cancer (9), renal cell carcinoma (10), prostate cancer (11), and lymphoma cells (12). In neuroblastoma, increased expression of the Ezh2 gene has been shown to enhance the proliferation, migration, and angiogenesis capacity of the tumour cells, and these phenotypes could be reduced by the downregulation of Ezh2 gene expression (13). These results indicate that the Ezh2 gene plays a key role in maintaining the growth and invasion of neuroblastoma. Studies have also shown that the apoptosis of glioma cells is closely related to the mitochondrial pathway $(14,15)$. In this study, we showed that the silencing of the Ezh2 gene leads to changes in the levels of Bax and $\mathrm{Bcl}-2$. The translocations of Bax and Bcl-2 have been shown to alter the mitochondrial membrane potential, cause the release of cytochrome $c$, activate the caspase family, and eventually lead to apoptosis (16).

We investigated the effects of the downregulation of the Ezh2 gene on the proliferation, apoptosis, and cell cycle of human glioma cells. We also explored the signal transduction pathway induced by the silencing of the Ezh2 gene that results in apoptosis.

\section{Materials and methods}

Cells, antibodies, and reagents. The U87 and U251 human glioma cells were purchased from the American Type Culture Collection (ATCC; USA). Lipofectamine ${ }^{\mathrm{TM}} 2000$ was purchased from Invitrogen (USA), the siRNAs were synthesised by GenePharma (China), the RT-PCR kit [Takara 
RNA PCR kit (AMV) Ver. 3.0] was purchased from Takara Biotechnology (Japan), and propidium iodide was purchased from Sigma (USA). The antibodies against Ezh2, Bax, Bcl-2, caspase 3 and 9, CDK4, CDK6, and cyclin D1 were purchased from Cell Signaling Technology, Inc., USA.

Cell culture. The U87 and U251 human glioma cells were cultured in Dulbecco's modified Eagle's medium (DMEM) containing $10 \%$ foetal bovine serum, $100 \mathrm{U} / \mathrm{ml}$ penicillin, and $100 \mu \mathrm{g} / \mathrm{ml}$ streptomycin at $37^{\circ} \mathrm{C}$ with $5 \% \mathrm{CO}_{2}$. All of the experiments were performed with logarithmically growing cells.

Detection of cellular viability. To measure the cellular viability using the MTT assay, the cells were seeded in 96-well culture plates at a density of $1 \times 10^{5}$ cells $/ \mathrm{ml}$, and Ezh2 siRNA was added when the cells were determined to be logarithmically growing. There were 5 groups of 6 wells of cells for each time point $(24,48,72,96$ and $120 \mathrm{~h})$ within each experiment. After $20 \mu \mathrm{l}$ of a $5-\mathrm{mg} / \mathrm{ml}$ solution of 5-diphenyltetrazolium bromide (MTT) was added to each well, the cells were cultured for an additional $4 \mathrm{~h}$. Then, the culture medium was removed, and $150 \mu \mathrm{l}$ of dimethyl sulphoxide (DMSO) was added to each well. The cells were subsequently vortexed at room temperature for $10 \mathrm{~min}$, and the OD value $(570 \mathrm{~nm})$ of each well was detected with a microplate reader.

siRNA transfection. For the siRNA transfections, the cells were seeded in 6-well plates at a density of $5 \times 10^{5}$ cells $/ \mathrm{ml}$ and cultured in DMEM without antibiotics. The transfection was performed when the confluence of the cells was $\geq 60 \%$, and 2 individual groups of cells were either transfected with an Ezh2 siRNA or a non-targeting oligonucleotide control using Lipofectamine (Invitrogen) according to the manufacturer's instructions. The media were changed prior to the transfection with serum-free DMEM without antibiotics and replaced 4-6 $\mathrm{h}$ after the transfection with serum-containing DMEM medium. The sequence of the Ezh2 siRNA was 5'-AAG ACT CTG AAT GCA GTT GCT-3', and a non-targeting siRNA served as a negative control. The efficiency of the siRNA was determined using reverse transcription polymerase chain reaction (RT-PCR) and western blotting.

RNA extraction and RT-PCR. The extraction of the total-RNA was performed using an RNAiso ${ }^{\mathrm{TM}}$ Plus kit (Takara) according to the manufacturer's instructions. After calculating the RNA concentration, the RT-PCR was performed using an RT-PCR kit (Takara) according to the product manual. The primers for the Ezh2 and $\beta$-actin RT-PCR reaction were synthesised by Invitrogen and were as follows: Ezh2, forward primer 5'-GCC AGA CTG GGA AGA AAT CTG-3' and reverse primer 5'-TGT GCT GGA AAA TCC AAG TCA-3'; $\beta$-actin, forward primer 5'-CTG GGA CGA CAT GGA GAA AA-3' and reverse primer 5'-AAG GAA GGC TGG AAG AGT GC-3'. The PCR reaction was then performed in a 50- $\mu 1$ volume with the following reaction conditions: an initial denaturing step at $94^{\circ} \mathrm{C}$ for $2 \mathrm{~min}$ and 30 cycles of denaturing at $94^{\circ} \mathrm{C}$ for $30 \mathrm{sec}$, annealing at $60^{\circ} \mathrm{C}$ for $30 \mathrm{sec}$, and extension at $72^{\circ} \mathrm{C}$ for $30 \mathrm{sec}$. The PCR products were separated on a $1.0 \%$ agarose gel by electrophoresis and analysed with a gel imaging scanning system.
Detection of apoptosis by flow cytometry. The cells were collected by trypsinisation and washed twice in ice-cold PBS. Next, the cells were resuspended in PBS, and a single-cell suspension was prepared by pipetting the medium up and down. An Annexin-V and PI staining solution was then added according to the manufacturer's instructions (Annexin-V-FITC kit, Biosea Biotechnology, China). The cells were stained for $15 \mathrm{~min}$ in the dark at room temperature, and the apoptotic cells were detected using a flow cytometer (Becton-Dickinson, USA).

Detection of mitochondrial membrane potential. JC-1 staining and flow cytometry were used to detect changes in the mitochondrial membrane potential according to a previously published protocol (17). The fluorescence signals of the JC-1 monomer and polymer were detected by the FL1 and FL2 detectors, respectively. FL1-H and FL2-H represent the green and red fluorescence intensities, respectively. CellQuest software was used for the quantification of the results.

Cell cycle analysis by flow cytometry. For the cell cycle analysis, the cells were trypsinised with $0.25 \%$ trypsin, collected, washed twice in PBS, and fixed with a $70 \%$ ethanol solution at $4^{\circ} \mathrm{C}$ overnight. The following day, the ethanol was discarded. Subsequently, the cells were washed twice with PBS, stained with $1 \mathrm{ml}$ of PI dye that contained $10 \mu \mathrm{g}$ of RNaseA and $5 \mu \mathrm{l}$ of Triton X-100 for $30 \mathrm{~min}$ at $4^{\circ} \mathrm{C}$ in the dark, and analysed by flow cytometry.

Western blotting. For the western blotting, the transfected U87 cells from each group were collected and washed twice in PBS. Then, $2 \mathrm{ml}$ of lysis buffer $(50 \mathrm{mM}$ Tris- $\mathrm{HCl} \mathrm{pH}$ 7.4, $137 \mathrm{mM}$ $\mathrm{NaCl}, 10 \%$ glycerol, $100 \mathrm{mM}$ sodium vanadate, $1 \mathrm{mM}$ PMSF, $10 \mathrm{mg} / \mathrm{ml}$ aprotinin, $10 \mathrm{mg} / \mathrm{ml}$ leupeptin, $1 \% \mathrm{NP}-40$, and $5 \mathrm{mM}$ cocktail) was added to the cells. The protein concentration was determined using the BCA method, and the proteins were stained with bromophenol blue. Equal amounts of protein were loaded and separated on a $10 \%$ polyacrylamide gel by SDS-PAGE electrophoresis, and the proteins were transferred onto a PVDF membrane using the semi-dry method. The membrane was then blocked with $5 \%$ non-fat dry milk overnight. The following day, the membrane was washed with TBST, incubated for $2 \mathrm{~h}$ with the primary antibodies, washed with TBST, and incubated for an additional $2 \mathrm{~h}$ with the secondary antibody. After adding the chemiluminescence reagent, X-ray autoradiography was performed, the bands were scanned, and the gray scale images were analysed.

Statistical analysis. SPSS 16.0 statistical software was used for the statistical analysis. The values are shown as the mean \pm SD. Statistical analysis was performed using the Student's t-test, and the differences between the groups were considered to be statistically significant at $\mathrm{p}<0.05$.

\section{Results}

The Ezh2 gene is highly expressed in U87 human glioma cells. The Ezh2 mRNA and protein expression levels in U87 and U251 human glioma cells were examined using RT-PCR and western blotting, respectively. We found that Ezh2 mRNA 

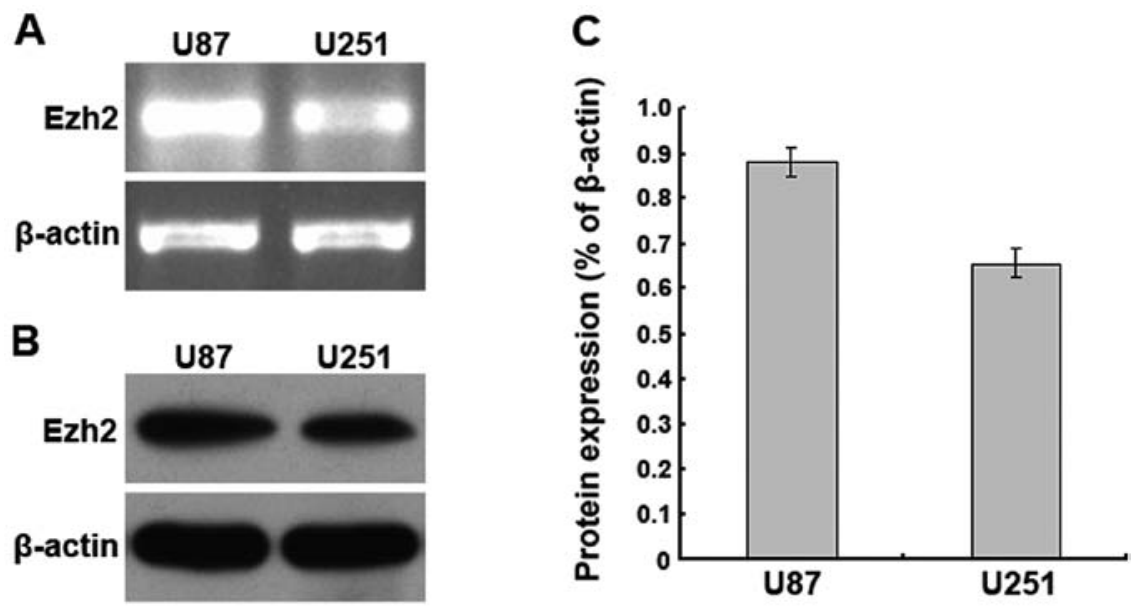

Figure 1. Ezh2 mRNA and protein expression levels in U87 and U251 cells. (A) The Ezh2 mRNA expression level as detected by RT-PCR. (B) Ezh2 protein expression level as detected by western blotting. (C) An analysis of the western blotting results with Gel-Pro Analyzer 4.0 software. The results shown are representative of 3 independent experiments.
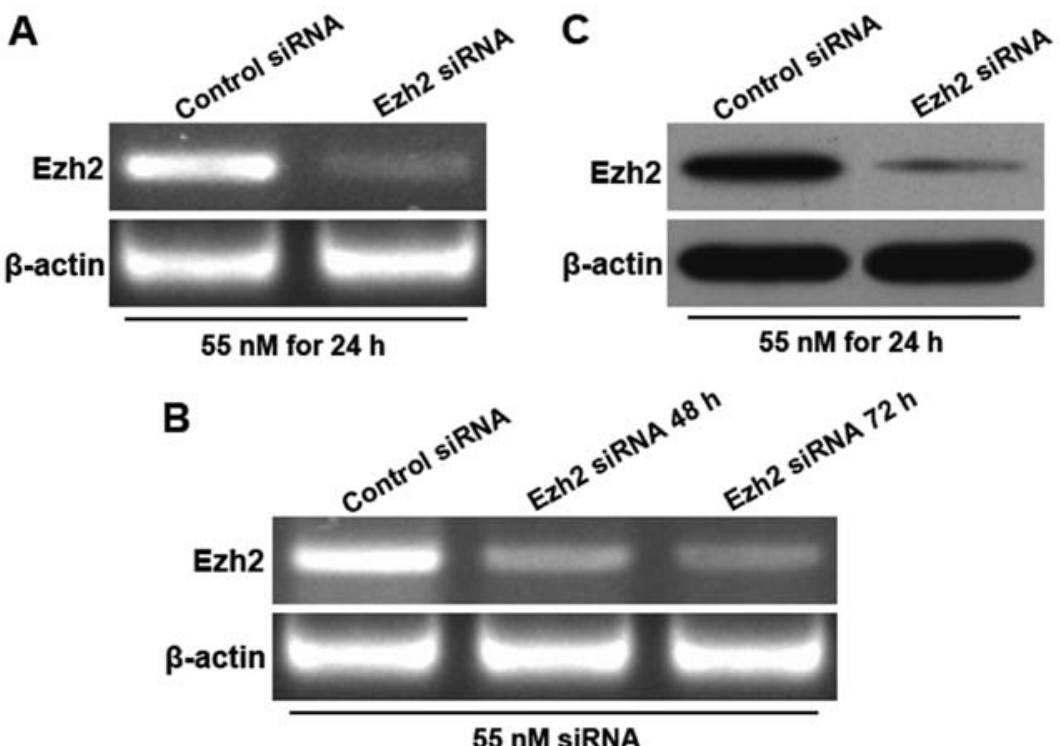

Figure 2. Ezh2 mRNA and protein expression levels in U87 cells after siRNA treatment. (A and B) After the U87 cells were transfected with siRNA targeting Ezh2, RT-PCR was used to determine the efficacy of the siRNA treatment. With a 55-nM siRNA transfection, the expression level of the Ezh2 mRNA was significantly reduced, and this reduction in mRNA expression lasted for at least $72 \mathrm{~h}$. (C) The Ezh2 siRNA efficacy was further analysed by western blotting. Twenty-four hours after the transfection with $55 \mathrm{nM}$ Ezh2 siRNA, the Ezh2 protein expression level was significantly reduced.

was highly expressed in the U87 and U251 cells, and a higher level was present in the U87 cells. Additionally, western blot analysis demonstrated that the expression levels of the Ezh2 protein in the U87 and U251 cells correlated with the mRNA expression levels. These results suggest that Ezh2 is highly expressed in the U87 and U251 human glioma cells (Fig. 1).

Downregulation of Ezh2 expression in U87 human glioma cells by RNA interference. An siRNA targeting Ezh2 and a non-targeting oligonucleotide were individually transfected into U87 cells, and the changes in the Ezh2 mRNA and protein expression levels after transfection were analysed by RT-PCR and western blotting, respectively. The results demonstrated that at $24 \mathrm{~h}$ after the transfection of the Ezh2 siRNA (55 nM), the Ezh2 mRNA expression level was significantly reduced.
The silencing of Ezh2 lasted for at least $72 \mathrm{~h}$ after the siRNA transfection (Fig. 2). These results suggest that after the transfection of the siRNA targeting Ezh2 at $55 \mathrm{nM}$ for $24 \mathrm{~h}$, the Ezh2 mRNA and protein expression levels are effectively downregulated.

Downregulation of Ezh2 expression inhibits the proliferation of U87 human glioma cells. MTT assay was used to determine the effects of the downregulation of Ezh2 expression on the proliferation of U87 cells. We found that compared to the mocktransfected cells or the cells transfected with the non-targeting siRNA, the proliferation of the U87 cells transfected with the Ezh2 siRNA was significantly reduced at $24,48,72,96$, or $120 \mathrm{~h}$ after the transfection. These results suggest that the silencing of the Ezh2 gene inhibits the proliferation of U87 cells (Fig. 3). 


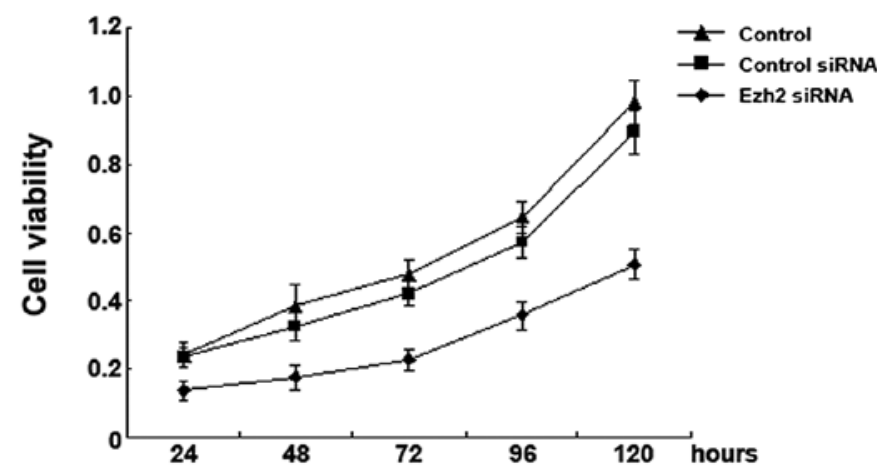

Figure 3. Downregulation of Ezh2 expression inhibits the proliferation of U87 cells. MTT assay was used to measure the proliferation of the U87 cells that had been transfected with either the Ezh2 siRNA or a non-targeting siRNA control. The proliferation level in the cells with downregulated Ezh2 expression was significantly reduced compared to the proliferation level in the mock-transfected cells or the cells that had been transfected with a nontargeting siRNA as a negative control. The results shown are representative of 3 independent experiments.

Downregulation of Ezh2 expression induces apoptosis in U87 human glioma cells. To investigate whether the level of Ezh2 expression was related to the level of apoptosis found in the U87 cells, the expression of the Ezh2 gene was downregulated by RNA interference, and the indicators of apoptosis were analysed. We found that after the silencing of the Ezh2 gene, a significantly higher rate of apoptosis was present in the U87 cells as detected by flow cytometry (Fig. 4A and B). In addition, western blot analysis indicated that the protein expression levels of procaspase 9 and 3 were significantly reduced after the downregulation of Ezh2 expression (Fig. 4C and D). These results suggest that the silencing of the Ezh2 gene leads to apoptosis in U87 cells.

Downregulation of Ezh2 expression induces apoptosis in the U87 human glioma cells through the mitochondrial pathway. To verify whether the apoptosis induced by the silencing of the Ezh2 gene in the U87 cells was related to the mitochondrial pathway, the changes in the mitochondrial membrane potential were detected using JC-1 staining. The results demonstrated that the downregulation of Ezh2 expression significantly reduced the mitochondrial membrane potential (Fig. 5A). In addition, the levels of $\mathrm{Bax}, \mathrm{Bcl}-2$, and cytochrome $c$ were analysed by western blotting. The silencing of Ezh2 expression decreased the level of Bax and increased the levels of Bcl-2 and cytochrome $c$ in the cytoplasm. However, the changes in the levels of $\mathrm{Bax}, \mathrm{Bcl}-2$, and cytochrome $c$ in the mitochondria were the opposite of the changes found in the cytoplasm (Fig. 5B-E). Therefore, our data indicate that the downregulation of Ezh2 expression promotes the translocation of $\mathrm{Bax}$ and $\mathrm{Bcl}-2$, causes the release of mitochondrial cytochrome $c$, reduces the mitochondrial membrane potential, and results in the apoptosis of human glioma cells.
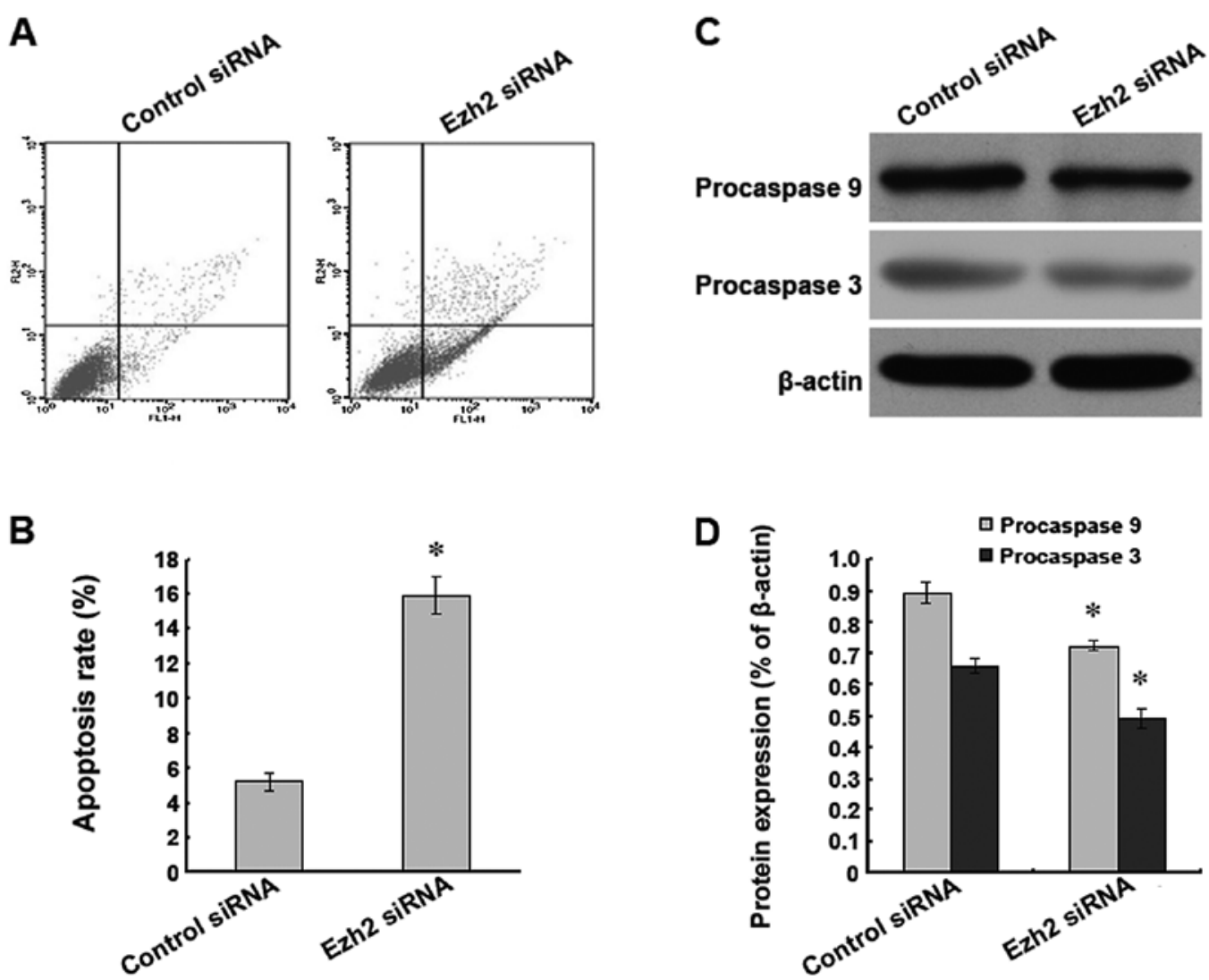

Figure 4. Downregulation of Ezh2 expression leads to apoptosis in U87 cells. (A) The apoptosis rate in U87 cells was analysed by flow cytometry. (B) A histogram representing the apoptosis rate in the U87 cells (\%). (C) The changes in the protein expression levels of procaspase 9 and 3 after Ezh 2 siRNA treatment were detected by western blotting. (D) The western blotting results were analysed using Gel-Pro Analyzer 4.0 software. ${ }^{*}$ p $<0.05$. The results shown are representative of 3 independent experiments. 
A

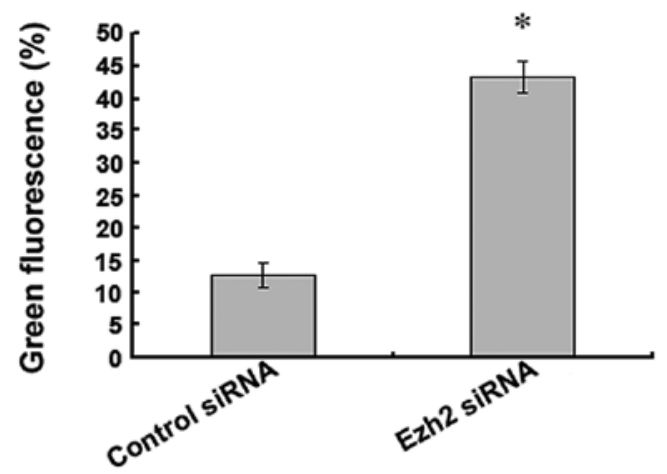

B
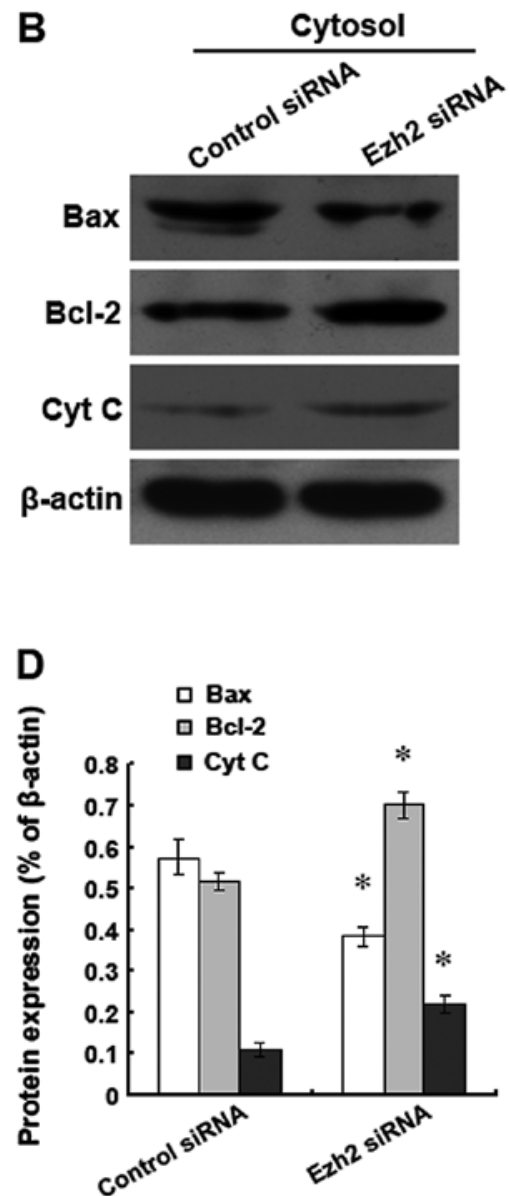

C
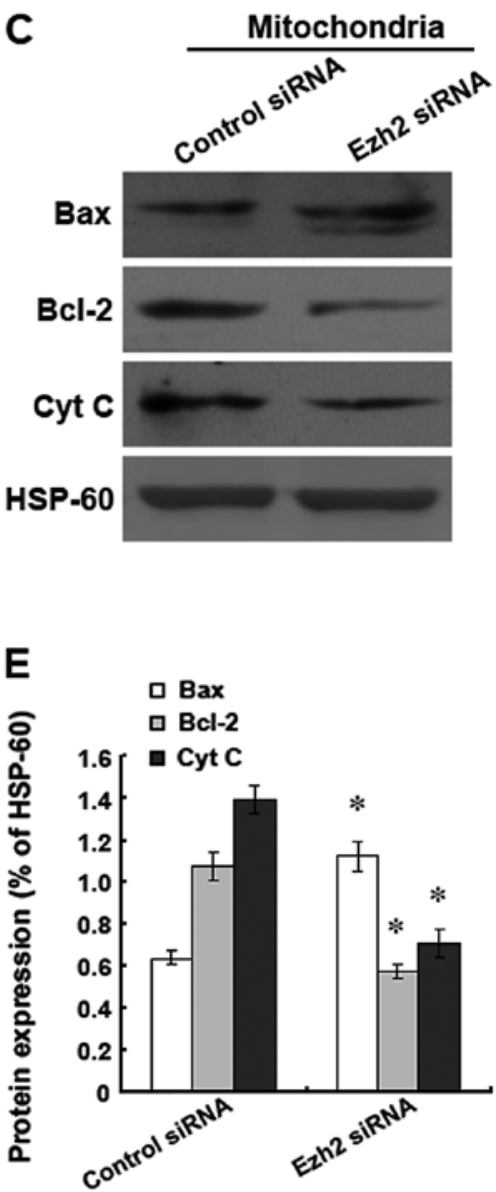

Figure 5. Downregulation of Ezh2 expression induces apoptosis in U87 cells through the mitochondrial pathway (A) Changes in the mitochondrial membrane potential were analysed by JC-1 staining and subsequent flow cytometry. (B and C) The expression levels of Bax, Bcl-2, and cytochrome $c$ in the cytoplasm and mitochondria were analysed by western blotting. (D and E) The western blotting results were analysed with Gel-Pro Analyzer 4.0 software. ${ }^{*}$ p $<0.05$. The results shown are representative of 3 independent experiments.

Downregulation of Ezh2 expression causes a cell cycle arrest in the G0/G1 phase in U87 human glioma cells. To further investigate the inhibition of proliferation caused by Ezh2 siRNA treatment, U87 cells were individually transfected with $55 \mathrm{nM}$ Ezh2 siRNA or a non-targeting siRNA as a negative control, and the cell cycle was analysed by flow cytometry. We found that upon Ezh2 siRNA treatment, the percentage of cells in the G0/G1 phase was significantly higher than the percentage found in the mock-transfected cells or the cells treated with the non-targeting siRNA (Fig. 6A). Additionally, we found that the downregulation of Ezh2 expression altered the levels of several cell cycle proteins, including cyclin D1, CDK4, and CDK6, all of which have roles in the G0/G1 phase. Western blot analysis revealed that compared with the mock-transfected cells or the cells transfected with the non-targeting siRNA, the protein expression levels of cyclin D1, CDK4, and CDK6 were significantly reduced in the cells transfected with the Ezh2 siRNA (Fig. 6B and C). These results suggest that the inhibition of proliferation caused by the downregulation of Ezh2 expression in the U87 cells may be mediated by the reduced expression levels of cyclin D1, CDK4, and CDK6, which result in an arrest of the cell cycle in the G0/G1 phase. 
A
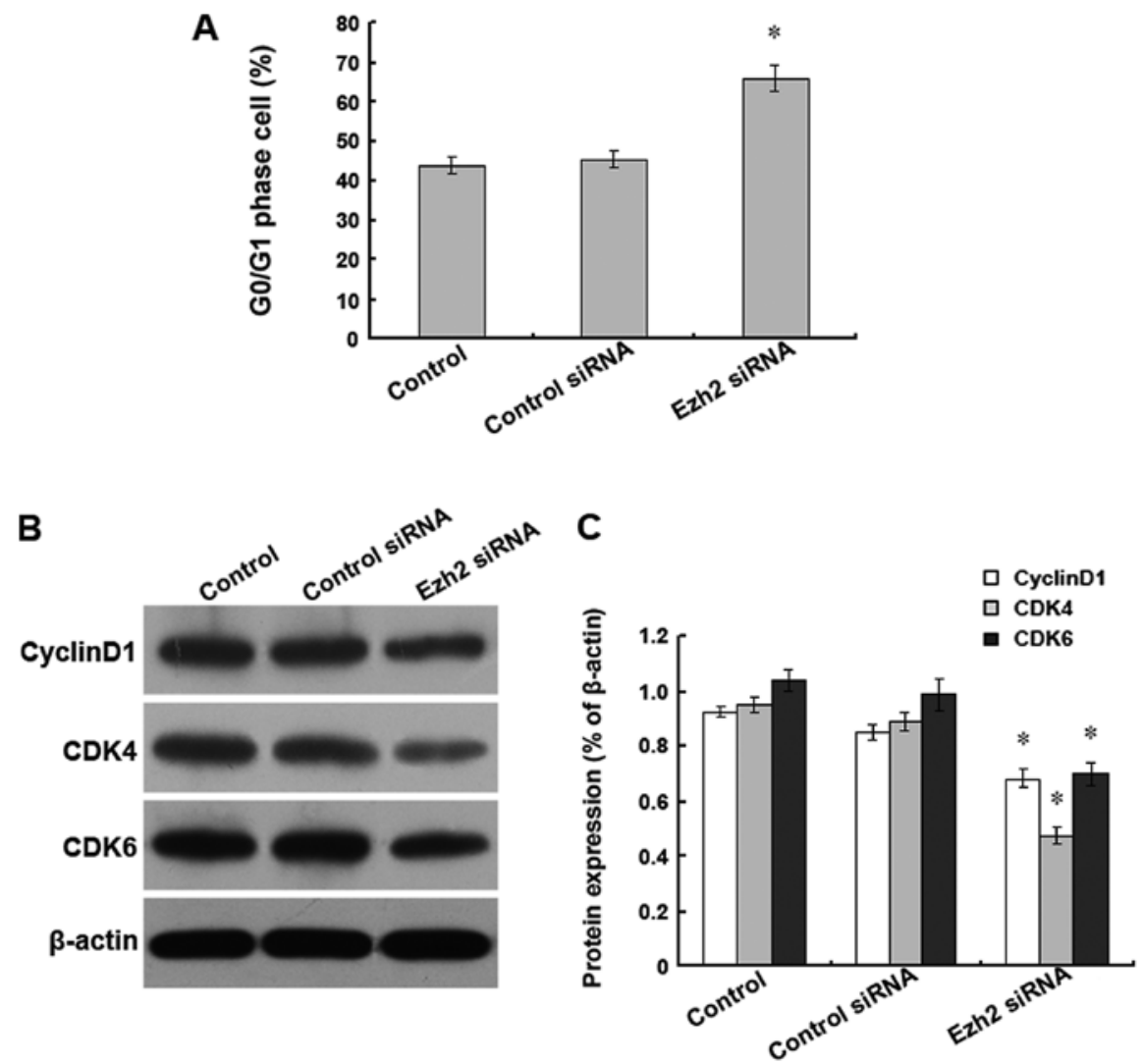

Figure 6. Downregulation of Ezh2 expression causes a cell cycle arrest in U87 cells in the G0/G1 phase. (A) The cell cycle distribution of U87 cells that had been treated with Ezh2 siRNA was analysed by flow cytometry. (B) The protein expression levels of cyclin D1, CDK4, and CDK6 were analysed by western blotting. (C) The western blotting results were examined using Gel-Pro Analyzer 4.0 software. "p $<0.05$. The results shown are representative of 3 independent experiments.

\section{Discussion}

In the present study, we explored the role of the Ezh2 gene for the treatment of human glioma. We demonstrated that Ezh2 was highly expressed both at the mRNA and protein level in human glioma cells, which suggests that the Ezh2 gene is closely related to the occurrence and development of glioma. Additionally, we hypothesised that the Ezh2 gene may serve as a potential biomarker and therapeutic target for the treatment of glioma. To further elucidate the detailed mechanism of the function of Ezh2 in human glioma, we used RNA interference to downregulate the expression of Ezh2 and observe the resulting changes in human glioma cells. We found that the Ezh2 mRNA and protein expression levels were significantly reduced with the siRNA treatment, and the downregulation of Ezh2 expression led to a reduction in the proliferation of the glioma cells. These data suggest that the Ezh2 gene plays a role in promoting the proliferation of glioma cells. Consistent with our findings, previous studies have also shown that the Ezh2 gene promotes the proliferation of lung (18), breast (9), thyroid (19), colon (20), ovarian (21), and pancreatic cancer cells (22). Therefore, we concluded that the Ezh2 gene plays an important role in the regulation of the proliferation of glioma cells.

To clarify how the downregulation of Ezh2 expression inhibited the proliferation of glioma cells, we used flow cytometry to analyse the cell cycle and determine the level of apoptosis. Our results demonstrated that the silencing of Ezh2 expression led to an induction of apoptosis in the glioma cells.
To date, research regarding the role of Ezh2 in the induction of apoptosis is very limited. One study demonstrated that the silencing of the Ezh2 gene led to increased levels of apoptosis in renal cancer cells (23). In addition, the suppression of Ezh2 expression has been reported to cause apoptosis by downregulating the expression levels of Bax and caspase 3 (24). Consistent with these studies, we also found that the reduced proliferation caused by Ezh2 siRNA treatment correlated with an increased level of apoptosis in the glioma cells. We also found that the downregulation of Ezh2 expression caused an arrest of the cell cycle in the G0/G1 phase and a reduction in the protein levels of the cell cycle regulatory proteins, cyclin D1, CDK4, and CDK6. These results indicate that the Ezh2 gene is essential for cellular proliferation, and the downregulation of Ezh2 expression leads to a reduced number of dividing glioma cells, which is consistent with previous reports $(25,26)$.

Tumour cells can undergo apoptosis through the mitochondrial pathway or the death receptor pathway (27). We found that in glioma cells, the silencing of Ezh2 expression led to the translocation of Bax and Bcl-2, which was followed by a decrease in the mitochondrial membrane potential and the release of cytochrome $c$. These results suggest that the apoptosis of glioma cells induced by the downregulation of Ezh2 expression is dependent on the mitochondrial pathway. Bcl-2 and Bax play key regulatory roles in the mitochondrial apoptosis pathway (28). When Bax translocates from the cytoplasm to the mitochondrial membrane, it changes the permeability of the mitochondrial membrane and promotes the release of cyto- 
chrome $c$ from the mitochondria into the cytoplasm (29) to initiate the events that lead to cellular apoptosis. The activation of the caspase family is necessary to induce apoptosis. Herein, we analysed the protein levels of procaspase 9 and procaspase 3 after Ezh2 siRNA treatment and found that the levels of these proteins were significantly reduced. Cytochrome $c$ released into the cytoplasm activates caspase 9 and caspase 3 , which play key roles in the apoptosis pathway (30). Therefore, these results suggest that the downregulation of Ezh2 expression induces apoptosis through the mitochondrial pathway in glioma cells.

Collectively, we demonstrated that the Ezh2 gene is highly expressed in human glioma cells, and the downregulation of Ezh2 expression induces apoptosis through the mitochondrial pathway by regulating the $\mathrm{Bcl}-2 / \mathrm{Bax}$ family in human glioma cells. Furthermore, this study provides a new approach for the clinical treatment of glioma.

\section{Acknowledgements}

We thank Professor Luo Yinan from the First Hospital of Jilin University and Professor Li Shulei from the College of Medicine of Jilin University for their guidance of this study.

\section{References}

1. Johannesen TB, Langmark F and Lote K: Cause of death and long-term survival in patients with neuro-epithelial brain tumours: a population-based study. Eur J Cancer 39: 2355-2363, 2003.

2. Hess KR, Broglio KR and Bondy ML: Adult glioma incidence trends in the United States, 1977-2000. Cancer 101: 2293-2299, 2004.

3. Stummer W and Kamp MA: The importance of surgical resection in malignant glioma. Curr Opin Neurol 22: 645-649, 2009.

4. Norden AD and Wen PY: Glioma therapy in adults. Neurologist 12: 279-292, 2006

5. De Haan G and Gerrits A: Epigenetic control of hematopoietic stem cell aging the case of Ezh2. Ann NY Acad Sci 1106: 233-239, 2007.

6. Venneti S, Le P, Martinez D, Xie SX, Sullivan LM, Rorke-Adams LB, Pawel B and Judkins AR: Malignant rhabdoid tumors express stem cell factors, which relate to the expression of EZH2 and Id proteins. Am J Surg Pathol 35: 1463-1472, 2011.

7. Juan AH, Derfoul A, Feng X, Ryall JG, Dell'Orso S, Pasut A, Zare H, Simone JM, Rudnicki MA and Sartorelli V: Polycomb EZH2 controls self-renewal and safeguards the transcriptional identity of skeletal muscle stem cells. Genes Dev 25: 789-794, 2011.

8. Yonemitsu Y, Imazeki F, Chiba T, Fukai K, Nagai Y, Miyagi S, Arai M, Aoki R, Miyazaki M, Nakatani Y, et al: Distinct expression of polycomb group proteins EZH2 and BMI1 in hepatocellular carcinoma. Hum Pathol 40: 1304-1311, 2009.

9. Reijm EA, Jansen MP, Ruigrok-Ritstier K, van Staveren IL, Look MP, van Gelder ME, Sieuwerts AM, Sleijfer S, Foekens JA and Berns EM: Decreased expression of EZH2 is associated with upregulation of ER and favorable outcome to tamoxifen in advanced breast cancer. Breast Cancer Res Treat 125: 387-394, 2011.

10. Hinz S, Weikert S, Magheli A, Hoffmann M, Engers R, Miller K and Kempkensteffen C: Expression profile of the polycomb group protein enhancer of Zeste homologue 2 and its prognostic relevance in renal cell carcinoma. J Urol 182: 2920-2925, 2009.

11. Varambally S, Dhanasekaran SM, Zhou M, Barrette TR, Kumar-Sinha C, Sanda MG, Ghosh D, Pienta KJ, Sewalt RG, Otte AP, et al: The polycomb group protein EZH2 is involved in progression of prostate cancer. Nature 419: 624-629, 2002.

12. Visser HP, Gunster MJ, Kluin-Nelemans HC, Manders EM, Raaphorst FM, Meijer CJ, Willemze R and Otte AP: The Polycomb group protein EZH2 is upregulated in proliferating, cultured human mantle cell lymphoma. Br J Haematol 112: 950-958, 2001.
13. Smits M, Nilsson J, Mir SE, van der Stoop PM, Hulleman E, Niers JM, de Witt Hamer PC, Marquez VE, Cloos J, Krichevsky AM, et al: miR-101 is down-regulated in glioblastoma resulting in EZH2-induced proliferation, migration, and angiogenesis. Oncotarget 1: 710-720, 2010.

14. Tomiyama A, Tachibana K, Suzuki K, Seino S, Sunayama J, Matsuda KI, Sato A, Matsumoto Y, Nomiya T, Nemoto K, et al: MEK-ERK-dependent multiple caspase activation by mitochondrial proapoptotic Bcl-2 family proteins is essential for heavy ion irradiation-induced glioma cell death. Cell Death Dis 1: e60, 2010.

15. Qiao S, Murakami K, Zhao Q, Wang B, Seo H, Yamashita H, Li X, Iwamoto T, Ichihara $M$ and Yoshino M: Mimosineinduced apoptosis in C6 glioma cells requires the release of mitochondria-derived reactive oxygen species and p38, JNK activation. Neurochem Res 37: 417-427, 2012

16. Lu HF, Chie YJ, Yang MS, Lee CS, Fu JJ, Yang JS, Tan TW, Wu SH, Ma YS, Ip SW and Chung JG: Apigenin induces caspasedependent apoptosis in human lung cancer A549 cells through Bax- and Bcl-2-triggered mitochondrial pathway. Int J Oncol 36: 1477-1484, 2010.

17. Tang B, Zhang Y, Liang R, Yuan P, Du J, Wang H and Wang L: Activation of the $\delta$-opioid receptor inhibits serum deprivationinduced apoptosis of human liver cells via the activation of PKC and the mitochondrial pathway. Int J Mol Med 28: 1077-1085, 2011.

18. Dang X, Ma A, Yang L, Hu H, Zhu B, Shang D, Chen T and Luo Y: MicroRNA-26a regulates tumorigenic properties of EZH2 in human lung carcinoma cells. Cancer Genet 205: 113-123, 2012.

19. Esposito F, Tornincasa M, Pallante P, Federico A, Borbone E, Pierantoni GM and Fusco A: Down-regulation of the miR-25 and miR-30d contributes to the development of anaplastic thyroid carcinoma targeting the polycomb protein EZH2. J Clin Endocrinol Metab 97: E710-E718, 2012.

20. Fussbroich B, Wagener N, Macher-Goeppinger S, Benner A, Fälth M, Sültmann H, Holzer A, Hoppe-Seyler K and HoppeSeyler F: EZH2 depletion blocks the proliferation of colon cancer cells. PLoS One 6: e21651, 2011.

21. Li H, Cai Q, Godwin AK and Zhang R: Enhancer of zeste homolog 2 promotes the proliferation and invasion of epithelial ovarian cancer cells. Mol Cancer Res 8: 1610-1618, 2010.

22. Ougolkov AV, Bilim VN and Billadeau DD: Regulation of pancreatic tumor cell proliferation and chemoresistance by the histone methyltransferase enhancer of zeste homologue 2. Clin Cancer Res 14: 6790-6796, 2008.

23. Wagener N, Holland D, Bulkescher J, Crnković-Mertens I, Hoppe-Seyler K, Zentgraf H, Pritsch M, Buse S, Pfitzenmaier J, Haferkamp A, et al: The enhancer of zeste homolog 2 gene contributes to cell proliferation and apoptosis resistance in renal cell carcinoma cells. Int J Cancer 123: 1545-1550, 2008.

24. Wu ZL, Zheng SS, Li ZM, Qiao YY, Aau MY and Yu Q: Polycomb protein EZH2 regulates E2F1-dependent apoptosis through epigenetically modulating Bim expression. Cell Death Differ 17: 801-810, 2010.

25. Wu SC and Zhang Y: Cyclin-dependent kinase 1 (CDK1)mediated phosphorylation of enhancer of zeste 2 (Ezh2) regulates its stability. J Biol Chem 286: 28511-28519, 2011.

26. Chen S, Bohrer LR, Rai AN, Pan Y, Gan L, Zhou X, Bagchi A, Simon JA and Huang H: Cyclin-dependent kinases regulate epigenetic gene silencing through phosphorylation of EZH2. Nat Cell Biol 12: 1108-1114, 2010.

27. von Haefen C, Wendt J, Semini G, Sifringer M, Belka C, Radetzki S, Reutter W, Daniel PT and Danker K: Synthetic glycosidated phospholipids induce apoptosis through activation of FADD, caspase- 8 and the mitochondrial death pathway. Apoptosis 16: 636-651, 2011.

28. Mattson MP and Kroemer G: Mitochondria in cell death: novel targets for neuroprotection and cardioprotection. Trends Mol Med 9: 196-205, 2003.

29. Saito M, Korsmeyer SJ and Schlesinger PH: BAX-dependent transport of cytochrome c reconstituted in pure liposomes. Nat Cell Biol 2: 553-555, 2000.

30. Riedl SJ and Shi Y: Molecular mechanisms of caspase regulation during apoptosis. Nat Rev Mol Cell Biol 5: 897-907, 2004. 